\title{
Self-Similarity Analysis of the Nonlinear Schrödinger Equation in the Madelung Form
}

\author{
Imre F. Barna $\mathbb{D}^{1,2}$ Mihály A. Pocsai, ${ }^{1,3}$ and L. Mátyás ${ }^{4}$ \\ ${ }^{1}$ Wigner Research Center for Physics of the Hungarian Academy of Sciences, Konkoly-Thege Miklós út 29-33, 1121 Budapest, Hungary \\ ${ }^{2}$ ELI-HU Nonprofit Kft., Dugonics Tér 13, H-6720 Szeged, Hungary \\ ${ }^{3}$ University of Pécs, Institute of Physics, Ifúság Útja 6, H-7624 Pécs, Hungary \\ ${ }^{4}$ Sapientia University, Department of Bioengineering, Libertătii Sq. 1, 530104 Miercurea Ciuc, Romania
}

Correspondence should be addressed to Imre F. Barna; barna.imre@wigner.mta.hu

Received 16 July 2018; Accepted 12 September 2018; Published 1 October 2018

Academic Editor: Emmanuel Lorin

Copyright (c) 2018 Imre F. Barna et al. This is an open access article distributed under the Creative Commons Attribution License, which permits unrestricted use, distribution, and reproduction in any medium, provided the original work is properly cited.

In the present study a particular case of Gross-Pitaevskii or nonlinear Schrödinger equation is rewritten to a form similar to a hydrodynamic Euler equation using the Madelung transformation. The obtained system of differential equations is highly nonlinear. Regarding the solutions, a larger coefficient of the nonlinear term yields stronger deviation of the solution from the linear case.

\section{Introduction}

In 1926, at the advent of the quantum mechanics Madelung $[1,2]$ gave a hydrodynamical foundation of the Schrödinger equation and raised interpretation questions which are open till today. Later, people realized that the Madelung Anzatz is just the complex Cole-Hopf transformation $[3,4]$ which is sometimes applied to linearize nonlinear partialdifferential equations (PDEs). The Madelung form of the Schrödinger equation has a remarkable property that Planck's constant (representing quantum mechanics) appears only once, namely, as the coefficient of the quantum potential. This quantity also appears in the nonmainstream attempt of quantum mechanics (the de Broglie-Bohm pilot wave theory [5] (in other context)) which interprets quantum mechanics as a deterministic nonlocal theory. The Madelung transformation may create a common language between scientist of fluid dynamics and quantum mechanics and can be fruitful to give an additional interpretation of quantum mechanics. In the last decades, hydrodynamical description of quantum mechanical systems become a popular technical tool in numerical simulations. Detailed reviews in this field can be found in a booklet [6] or in Wyatt's monograph [7].

The application of the original Madelung (or to say ColeHopf) transformation grew out the linear quantum theory and were applied to more sophisticated field theoretical problems. Recently, Chavanis and Matos [8] applied the Madelung Ansatz to the Klein-Gordon-Maxwell-Einstein equation in curved space-time possibly describing selfgravitating Bose-Einstein condensates, coupled to an electromagnetic field. This study clearly presents the wide applicability of the original Madelung Ansatz to refined quantum field theories with the possible hydrodynamic interpretation picture.

As an analytical tool of investigation we use the selfsimilar Ansatz of [9]. It is a powerful method to study the global properties of the dispersive solutions of various nonlinear partial-differential equations (PDEs) In other words, the intermediate asymptotic of the problem is described. Therefore this solution is valid when the precise initial conditions are no longer important; however, the final steady state is not yet reached. For real physical systems this region attracts the most attention. In contrast to the full solution it is much simpler and easier to understand for the entire parameter space. The second reason for studying them is that they are solutions of a system of ordinary differential equations (ODEs) (not a PDE) and thus do not suffer the full inherent numerical problems of the full PDEs. Most of the cases this tool helps to understand some of the global properties of the solution, e.g., shock waves or the existence of 
compact supports. First, we applied this Ansatz to the modified Cattaneo-Venotte heat-conduction problem [10] and obtained a compact solution with finite propagation speed. Later, we successfully generalized the Ansatz to multispace dimensions and studied numerous viscous fluid equations $[11,12]$ ending up with a book chapter of [13]. As main results we are now able to interpret the birth of Benard cells in heated fluids [14].

In our former study [15] we investigated the Schrödinger equation in the Madelung form with the self-similar Ansatz and presented analytic solutions with discussion. Unlike to classical fluids the obtained density of the Madelung fluid has infinite number of zeroes which is a fingerprint of the quantum mechanical origin. Now we analyze the Madelung form of nonlinear Schrödinger (NLS) equation with the same Ansatz and compare the obtained results to the previous linear one. As far as we know, there are no direct clearcut analytic solutions existing for the Madelung form of the NLS equation by now. It is important to mention that celebrated standard numerical algorithms exist for solving the NLS $[16,17]$. We hope that our present study helps to attract attention to this question. Parallel to the well-known quantum mechanical picture there is a looming hydrodynamical world hidden behind the Schrödinger equation from the very beginning which is poorly understood.

\section{Theory and Results}

In a weakly interacting Bose system, the macroscopic wave function $\Psi$ appears as the order parameter of the BoseEinstein condensation, obeying the Gross-Pitaevskii (GP) equation [18-21]:

$$
i \hbar \frac{\partial \Psi}{\partial t}=\left(-\frac{\hbar^{2} \nabla^{2}}{2 m}+n|\Psi|^{2}-\mu\right) \Psi
$$

Here, $n=4 \pi \hbar^{2} a / m$ represents the coupling constant characterized by the s-wave scattering length a. The particle mass and the chemical potential are $m$ and $\mu$, respectively. Due to the second (nonlinear) term of the right hand side of this equation, it is definitely different from the linear Schrödinger equation [15]; therefore the following analysis with the presented results are independent as well.

The role and the properties of the GP system has been investigated from various viewpoints. Without completeness we mention some of them. The question of collective excitations of the trapped Bose gases was analyzed by [22]. Later, it is considered in the study of self-organization of the BoseEinstein (BE) condensates [23].

Certain generalized versions of (1) have been also applied to the study of BE condensate of polaritons [24-32].

Considering the Madelung Ansatz for the order parameter with the amplitude and phase $\Psi=\sqrt{\rho} e^{i \phi}$ where the density of the condensate is $\rho$ and the superfluid velocity is $\mathbf{v}=(\hbar / m) \nabla \phi$. (The expression "superfluid velocity" is taken from Tsubota et al. [33] and means, in this content, that the fluid is inviscid.)
The obtained hydrodynamical equations, the continuity and the Euler, are as follows:

$$
\begin{aligned}
& \frac{\partial \rho}{\partial t}+\nabla \cdot(\rho \mathbf{v})=0 \\
& \frac{\partial \mathbf{v}}{\partial t}+\mathbf{v} \cdot \nabla \mathbf{v}=\frac{\hbar^{2}}{2 m^{2}} \nabla\left(\frac{\triangle \sqrt{\rho}}{\sqrt{\rho}}\right)-\frac{\nabla}{m \rho}\left(\frac{n \rho^{2}}{2}\right),
\end{aligned}
$$

where we consider free motion (no extra potential energy term $U(\mathbf{r})$ is used) and the chemical potential $\mu$ was set to zero as well. It is easy to show that an additional chemical potential term $\mu$ gives no contribution to the final hydrodynamical equations. The role of the external potential energy term $U(\mathbf{r})$ will be mentioned later.

The first term of the right hand side of the second equation is the quantum pressure term. The second term makes the difference to the original Madelung equations; this is a kind of second quantum pressure due to the nonlinear origin of the GP equation. Not, that these are most general vectorial equation for the velocity field $\mathbf{v}$ which means that one-, two-, or three-dimensional motions can be investigated as well. In the following we will consider the two-dimensional flow motion $\mathbf{v}=(u, v)$ in Cartesian coordinates. The functional form of the three- and one-dimensional equation system will be mentioned briefly, too.

We search the solution of (2) in the form of the self-similar Ansatz [9]:

$$
\begin{aligned}
& \rho(x, y, t)=t^{-\alpha} f\left(\frac{x+y}{t^{\beta}}\right):=t^{-\alpha} f(\eta), \\
& u(x, y, t)=t^{-\delta} g(\eta), \\
& v(x, y, t)=t^{-\epsilon} h(\eta),
\end{aligned}
$$

where $f, g$, and $h$ are the shape functions of the density and the velocity field, respectively. The similarity exponents $\alpha, \beta, \delta, \epsilon$ are of primary physical importance since $\alpha, \delta, \epsilon$ represent the damping of the magnitude of the shape function. The exponent $\beta$ represents the spreading of the solution. Additional technical details about the general features of the Ansatz can be found in our former papers [12, 14]. Except some extraordinary cases only all positive similarity exponents correspond to physically relevant dispersive solutions which mean decaying features at $x, y, t \longrightarrow \infty$. Usually negative exponents describe exploding solutions. Substituting the Ansatz (3) into (2) and executing additional algebraic manipulation the following ODE system can written down for the shape functions:

$$
\begin{aligned}
& -\alpha f-\frac{\alpha}{2} f^{\prime} \eta+f^{\prime} g+f g^{\prime}+f^{\prime} h+f h^{\prime}=0, \\
& -\left(\frac{3}{2} \alpha-1\right) g-\frac{\alpha}{2} g^{\prime} \eta+g g^{\prime}+h g^{\prime} \\
& =\frac{\hbar^{2}}{2 m^{2}}\left(\frac{f^{\prime 3}}{2 f^{3}}-\frac{f^{\prime} f^{\prime \prime}}{f^{2}}+\frac{f^{\prime \prime \prime}}{2 f}\right)-\frac{n}{m} f^{\prime},
\end{aligned}
$$




$$
\begin{aligned}
& -\left(\frac{3}{2} \alpha-1\right) h-\frac{\alpha}{2} h^{\prime} \eta+g h^{\prime}+h h^{\prime} \\
& =\frac{\hbar^{2}}{2 m^{2}}\left(\frac{f^{\prime 3}}{2 f^{3}}-\frac{f^{\prime} f^{\prime \prime}}{f^{2}}+\frac{f^{\prime \prime \prime}}{2 f}\right)-\frac{n}{m} f^{\prime},
\end{aligned}
$$

where prime means derivation with respect to the new variable $\eta$. The relations among the similarity exponents are the following: $\alpha$ can be taken arbitrary, $\beta=\alpha / 2$ and $\epsilon=$ $\delta=3 \beta-1=3 \alpha / 2-1$. At this point we have to emphasize, that a particular form of the external potential $U(x, y, t)$ can be added to (1) without leading to contradiction among the similarity exponents. The form in $2 \mathrm{D}$ is the following: $U(x, y, t)=1 / t^{2 \beta} \cdot v(\eta)$ where $v(\eta)$ can be any kind of function with existing first derivative.

(Of course, there is no evidence that the obtained modified (4) could be solved with analytical means.)

Note, that the ODE system of the linear Schrödinger equation [15] has a much simpler form of

$$
\begin{aligned}
& -\frac{1}{2} f-\frac{1}{2} f^{\prime} \eta+f^{\prime} g+f g^{\prime}+f^{\prime} h+f h^{\prime}=0, \\
& -\frac{1}{2} g-\frac{1}{2} g^{\prime} \eta+g g^{\prime}+h g^{\prime} \\
& =\frac{\hbar^{2}}{2 m^{2}}\left(\frac{f^{\prime 3}}{2 f^{3}}-\frac{f^{\prime} f^{\prime \prime}}{f^{2}}+\frac{f^{\prime \prime \prime}}{2 f}\right), \\
& -\frac{1}{2} h-\frac{1}{2} h^{\prime} \eta+g h^{\prime}+h h^{\prime} \\
& =\frac{\hbar^{2}}{2 m^{2}}\left(\frac{f^{\prime 3}}{2 f^{3}}-\frac{f^{\prime} f^{\prime \prime}}{f^{2}}+\frac{f^{\prime \prime \prime}}{2 f}\right),
\end{aligned}
$$

where all the similarity exponents have the fixed value of $+1 / 2$. Our experience tells that this is usual for regular heat conduction, diffusion or for noncompressible Navier-Stokes equations [13]. For the linear case after some additional trivial algebraic steps a decoupled ODE can be derived for the shape function of the density:

$$
2 f^{\prime \prime} f-\left(f^{\prime}\right)^{2}+\frac{m^{2} \eta^{2} f^{2}}{2 \hbar^{2}}=0 .
$$

For one, two, or three dimensions the multiplicative factor of $\hbar$ in the last term is different. It is 1,2 , or 3 , respectively.

With the help of Bessel functions [34] the solution of (6) can be expressed:

$$
\begin{aligned}
& f(\eta) \\
& \quad=\frac{\pi \eta}{64}\left[c_{1} J_{1 / 4}\left(\frac{\sqrt{2} m \eta^{2}}{8}\right)-c_{2} Y_{1 / 4}\left(\frac{\sqrt{2} m \eta^{2}}{8}\right)\right]^{2},
\end{aligned}
$$

where $c_{1}$ and $c_{2}$ are the usual integration constants. All the additional properties of this solution can be found in our former paper [15]. (There is a misprint in the final [15] at Eq. 7 , the parenthesis is missing in front of the exponent 2 . This is the correct form.)
The most general equation of (4) cannot be fully integrated by analytical means. The first ODE of such systems [13] explicitly means mass conservation. Furthermore in the most cases it can be integrated analytically. However, the first equation of (4) is not a total derivative for any $\alpha$. This is highly unusual among our investigated systems till now. As a second case let us consider $(3 / 2 \alpha-1)=\alpha / 2$ this gives $\alpha=1$, $\beta=\delta=\epsilon=1 / 2$. Now the sum of the second and third equation of (5) can be integrated leading to

$$
\begin{aligned}
\frac{g^{2}}{2}+ & g\left(h-\frac{\eta}{2}\right)+\frac{h^{2}}{2}+\frac{h \eta}{2}+\frac{2 n f}{m} \\
& -\frac{\hbar^{2}}{2 m^{2}}\left(-\frac{f^{\prime 2}}{4 f^{2}}+\frac{f^{\prime \prime}}{2 f}\right)+c_{1}=0 .
\end{aligned}
$$

For the sake of simplicity we set $c_{1}$ to zero. Note, that the variables $g, f$ and $h$ are still coupled in this single equation. However, (8) is quadratic in $g(\eta)$ and the solutions can be expressed with the well-know formula of

$$
g_{1,2}=\frac{\eta}{2}-h \pm \sqrt{\frac{\eta^{2}}{4}-\frac{4 n f}{m}+\frac{\hbar^{2}}{2 m^{2}}\left(-\frac{f^{\prime 2}}{4 f^{2}}+\frac{f^{\prime \prime}}{2 f}\right)} .
$$

Even this includes both functions of $h$ and $f$. To avoid this problem let us fix the discriminant to zero, which means that the velocity shape function will be single valued. The remaining ODE reads as follows:

$$
2 f f^{\prime \prime}-\left(f^{\prime}\right)^{2}+f^{2}\left(\frac{\eta^{2} m^{2}}{2 \hbar^{2}}-\frac{8 n m f}{\hbar}\right)=0
$$

with the additional restraint that $4 f^{2} \neq 0$ meaning that the physical density of a fluid must be positive. Note, that without the last term we get back (6) with the solution of (7). We tried numerous variable transformations unfortunately, we could not find any closed solution for the full equation (10). (Note that if the coefficient of the second term would be twice as the first term there would be a possible solution for the full equation including the third order term.) It is also clear from (9) that the velocity shape functions have the trivial form of $g+h=\eta / 2=(x+y) / 2 \sqrt{t}$ which is the same as for the linear case.

Figure 1 shows the analytical solution of (7) and numerically solution of Eq. (10) for various nonlinear parameters $n$ with the same initial conditions of $f(0)=1$ and $f^{\prime}(0)=$ 0 . The $\hbar=m=1$ units are used. All solutions have a strong damping with stronger and stronger oscillations at large arguments. The functions are positive for all values of the argument, (which is physical for a fluid density), but such oscillatory profiles are completely unknown in regular fluid mechanics [13]. The most interesting feature is the infinite number of zero values which cannot be interpreted physically for a classical real fluid. Remembering the restraint $4 f^{2} \neq 0$ of the calculation the solution function falls into infinite number of finite intervals. We think that this is a clear fingerprint that the obtained Euler equations have a quantum mechanical origin. 


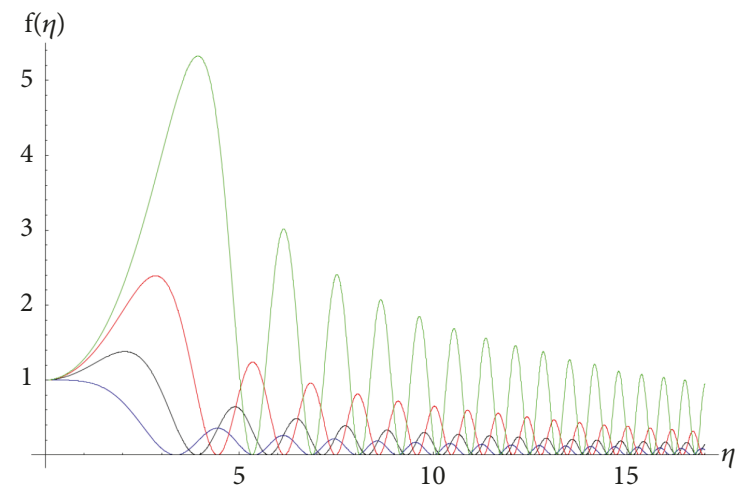

FIgURE 1: Various shape functions of the density. The blue curve is the analytic solution of equation (7) with $n=0$ nonlinearity parameter. Black, red, and green functions are numerical results of equation (10) for $n=0.078,0.11$ and 0.13 nonlinearity parameters. (All curves are for the same initial conditions of $f(0)=1$ and $f^{\prime}(0)=0$.) Note that larger parameters mean larger deviation from the analytic result.

The major difference to the analytic solution is the following the $\int_{0}^{\infty} f(\rho) d \rho$ can be evaluated (now of course just numerically up to an arbitrary $\rho_{\max }$ ) giving us a convergent integral. This clearly shows that the wave function of the corresponding quantum mechanical system (see below) is the element of the $L^{2}$ space. We investigated numerous initial conditions all giving the same result of a finite integral. Of course, this is just an empirical statement and not a rigorous mathematical proof.

The $\sqrt{\rho}$ function could be interpreted as the fluid mechanical analogue of the real part of the wave function of the free GP quantum mechanical particle. For the linear case the square root of (7) is a kind of far analogue of the original free Gaussian wave packet. Unfortunately, we cannot find any direct transformation between these two functions.

To obtain the complete original wave function, the imaginary part has to be evaluated as well. It is simple from $\eta=$ $(x+y) / t^{1 / 2}=2(g+h)$ that

$$
S=\frac{m}{\hbar} \int_{\mathbf{r}_{0}}^{\mathbf{r}_{1}} \mathbf{v d r}=\frac{m}{\hbar} \frac{(x+y)^{2}}{4 t} .
$$

Now

$$
\begin{aligned}
\Psi(x, y, t) & =\sqrt{\rho(x, y, t)} e^{i S(x, y, t)} \\
& =\sqrt{t^{-1} \cdot f\left(\frac{[x+y]}{t^{1 / 2}}\right)} e^{(i m / \hbar)\left((x+y)^{2} / 4 t\right)},
\end{aligned}
$$

where $f(\eta)$ is now a numerical function. Figure 2 shows the projection of the real part of the wave function to the $x, t$ subspace. At small times there are clear oscillations, but at larger times the strong damping is evident. If the time is fixed ( $t=t_{0}$ so to say) there are infinite number of spatial oscillations which can be interpreted as a quantum mechanical heritage of the system.

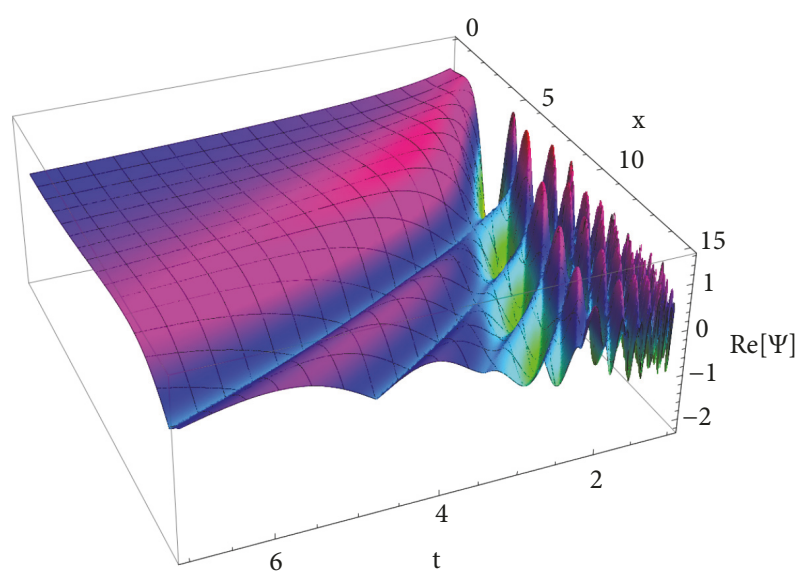

FIgURE 2: The projection of the real part of the wave function (12) along the $\mathrm{x}$-t plane.

\section{Summary and Outlook}

We gave a short historical review of the interpretation and development of the Madelung equation. As second point we introduced (the not so well known) self-similar Ansatz which is a robust tool to investigate the local and global properties of nonlinear PDEs. Finally, the Madelung form of the free NLS equation was investigated and the results were compared to the ones obtained from the linear Schrödinger equation. We think that the highly oscillatory fluid density (with the infinite number of zeroes) are a clear fingerprint of the quantum mechanical origin of the system. We also believe that the noticeable quadratic argument of the solutions might get an interpretation in the future. However, the presented approach is still evolving and works are in progress. We plan to apply our method to the Madelung equations of more general nonlinear Schrödinger equations like [35].

\section{Data Availability}

No data were used to support this study.

\section{Conflicts of Interest}

The authors declare that they have no conflicts of interest.

\section{Acknowledgments}

The ELI-ALPS project (GINOP-2.3.6-15-2015-00001) is supported by the European Union and cofinanced by the European Regional Development Fund.

\section{References}

[1] E. Madelung, "Eine anschauliche Deutung der Gleichung von Schrödinger," Naturwissenschaften, vol. 14, no. 45, p. 1004, 1926.

[2] E. Madelung and Z. Phys, "Quantentheorie in hydrodynamischer form," Zeitschrift für Physik, vol. 40, p. 332, 1927.

[3] E. Hopf, "The partial differential equation $U_{x}+U U_{t}=U_{x x}$ ", Communications on Pure and Applied Mathematics, vol. 3 , no. 3, pp. 201-230, 1950. 
[4] J. D. Cole, "On a quasi-linear parabolic equation occurring in aerodynamics," Quarterly of Applied Mathematics, vol. 9, no. 3, pp. 225-236, 1951.

[5] D. Bohm, "A suggested interpretation of the quantum theory in terms of hidden variables, I and II," Physical Review A: Atomic, Molecular and Optical Physics, vol. 85, pp. 166-193, 1952.

[6] K. H. Hughes and G. Parlant, Quantum Trajectories, CCP6, Daresbury Laboratory, 2011, http://www.ccp6.ac.uk/booklets/ CCP6-2011Q_Quantum_Trajectories.pdf.

[7] R. E. Wyatt, Quantum Dynamics with Trajectories Introduction to Quantum Hydrodynamics, vol. 28 of Interdisciplinary Applied Mathematics, Springer-Verlag, New York, 2005.

[8] P.-H. Chavanis and T. Matos, "Covariant theory of BoseEinstein condensates in curved spacetimes with electromagnetic interactions: The hydrodynamic approach," The European Physical Journal Plus, vol. 132, p. 30, 2017.

[9] L. I. Sedov, Similarity and Dimensional Methods in Mechanics, Mir Publishers, Moscow, Russia, 1993.

[10] I. F. Barna and R. Kersner, "Heat conduction: a telegraph-type model with self-similar behavior of solutions," Journal of Physics A: Mathematical and General, vol. 43, no. 37, 375210, 6 pages, 2010.

[11] I. F. Barna, "Self-similar solutions of three-dimensional navier-stokes equation," Communications in Theoretical Physics, vol. 56, p. 745, 2011.

[12] I. F. Barna and L. Mátyás, "Analytic solutions for the threedimensional compressible Navier-Stokes equation," Fluid Dynamics Research. An International Journal, vol. 46, no. 5, Article ID 055508, 10 pages, 2014.

[13] D. Campos, Handbook on Navier-Stokes Equations, Theory and Applied Analysis, Chapter 16, Nova Publishers, New York, NY, USA, 2017.

[14] I. F. Barna and L. Mátyás, "Analytic self-similar solutions of the Oberbeck-Boussinesq equations," Chaos, Solitons \& Fractals, vol. 78, pp. 249-255, 2015.

[15] I. F. Barna, M. Pocsai, L. Mátyás, and J. Gen, "Self-similarity analysis of the non-linear Schrödinger equation in the Madelung form," Journal of Generalized Lie Theory and Applications, vol. 11, p. 271, 2017.

[16] P. Muruganandam and S. K. Adhikari, "Fortran programs for the time-dependent Gross-Pitaevskii equation in a fully anisotropic trap," Computer Physics Communications, vol. 180, no. 10, pp. 1888-1912, 2009.

[17] D. Vudragović, I. Vidanović, A. Balaž, P. Muruganandam, and S. K. Adhikari, "C programs for solving the time-dependent Gross-Pitaevskii equation in a fully anisotropic trap," Computer Physics Communications, vol. 183, no. 9, pp. 2021-2025, 2012.

[18] E. P. Groos, "Structure of a quantized vortex in boson systems," Il Nuovo Cimento (1955-1965), vol. 20, pp. 454-477, 1961.

[19] L. P. Pitaevskii, "Vortex lines in an imperfect bose gas," Soviet Physics JETP, vol. 13, p. 451, 1961.

[20] F. Dalfovo, S. Giorgini, L. P. Pitaevskii, and S. Stringari, “Theory of Bose-Einstein condensation in trapped gases," Reviews of Modern Physics, vol. 71, no. 3, pp. 463-512, 1999.

[21] L. Erdös, B. Schlein, and H.-T. Yau, "Derivation of the GrossPitaevskii equation for the dynamics of Bose-Einstein condensate," Annals of Mathematics: Second Series, vol. 172, no. 1, pp. 291-370, 2010.

[22] A. Csordás and Z. Adam, "Collective excitations of trapped Fermi or Bose gases," Physical Review A: Atomic, Molecular and Optical Physics, vol. 74, Article ID 035602, 2006.
[23] D. Nagy, G. Szirmai, and P. Domokos, "Self-organization of a Bose-Einstein condensate in an optical cavity," The European Physical Journal D, vol. 48, p. 127, 2008.

[24] M. Wouters and I. Carusotto, "Excitations in a nonequilibrium bose-einstein condensate of exciton polaritons," Physical Review Letters, vol. 99, no. 14, 2007.

[25] K. G. Lagoudakis, M. Wouters, M. Richard et al., "Quantized vortices in an exciton-polariton condensate," Nature Physics, vol. 4, no. 9, pp. 706-710, 2008.

[26] G. Roumpos, M. Fraser, A. Löffler, S. Löffling, A. Forchel, and Y. Yamamoto, "Single vortex-antivortex pair in an excitonpolariton condensate," Nature Physics, vol. 7, pp. 129-133, 2011.

[27] E. A. Ostrovskaya, J. Abdullaev, A. S. Desyatnikov, M. D. Fraser, and Y. S. Kivshar, "Dissipative solitons and vortices in polariton Bose-Einstein condensates," Physical Review A: Atomic, Molecular and Optical Physics, vol. 86, no. 1, 2012.

[28] A. S. Rodrigues, P. G. Kevrekidis, R. Carretero-Gonzales, J. Cuevas-Maraver, and D. J. Frantzskakis, "From nodeless clouds and vortices to gray ring solitons and symmetry-broken states in two-dimensional polariton condensates," Journal of Physics: Condensed Matter, vol. 26, no. 15, Article ID 155801, 2014.

[29] V. S. Bagnato, D. J. Frantzeskakis, P. G. Kevrekidis, B. A. Malomed, and D. Mihalache, "Bose-Einstein condensation: twenty years after," Romanian Reports in Physics, vol. 67, no. 1, pp. 5-50, 2015.

[30] T. C. H. Liew, O. A. Egorov, M. Matuszewski, O. Kyriienko, X. $\mathrm{Ma}$, and E. A. Ostrovskaya, "Instability-induced formation and nonequilibrium dynamics of phase defects in polariton condensates," Physical Review B, vol. 91, 2015.

[31] A. M. Belounis and S. Kessal, "Shock wave occurrence and soliton propagation in polariton condensates," Canadian Journal of Physics, vol. 95, pp. 1234-1238, 2017.

[32] C. Schneider, K. Winkler, M. D. Fraser et al., "Exciton-polariton trapping and potential landscape engineering," Reports on Progress in Physics, vol. 80, no. 1, Article ID 016503, 2017.

[33] M. Tsubota, M. Kobayashi, and H. Takeuchi, "Quantum hydrodynamics," Physics Reports, vol. 522, no. 3, pp. 191-238, 2013.

[34] F. W. J. Olver, D. W. Lozier, R. F. Boisvert, and C. W. Clark, NIST Handbook of Mathematical Functions, Cambridge University Press, 2010.

[35] R. Carles, R. Danchin, and J.-C. Saut, "Madelung, gross-pitaevskii and korteweg," Nonlinearity, vol. 25, no. 10, pp. 28432873,2012 


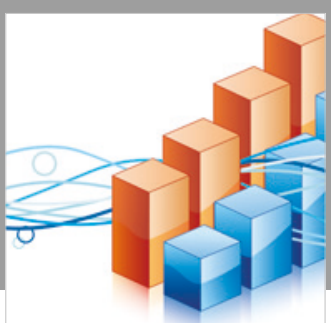

Advances in

Operations Research

\section{-n-m}
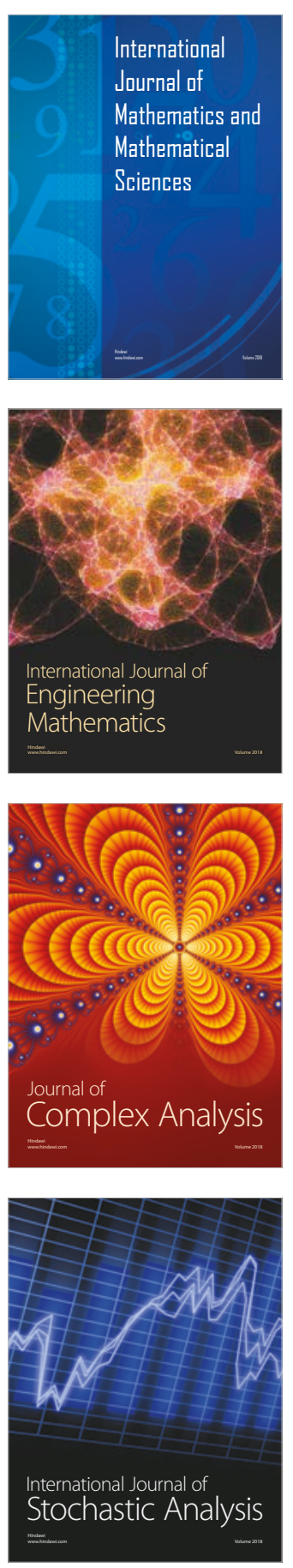
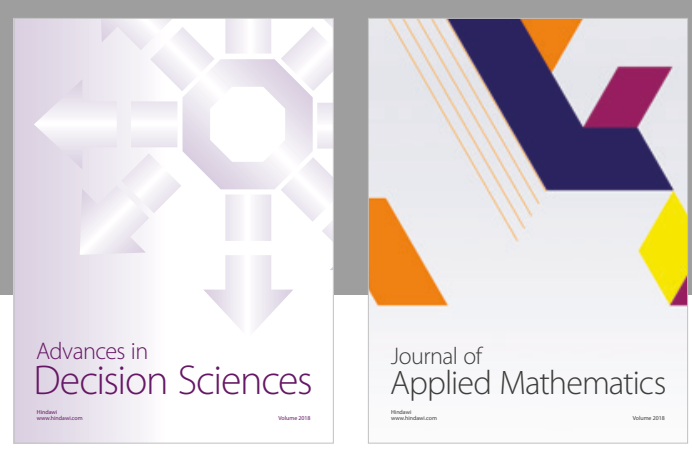

Journal of

Applied Mathematics
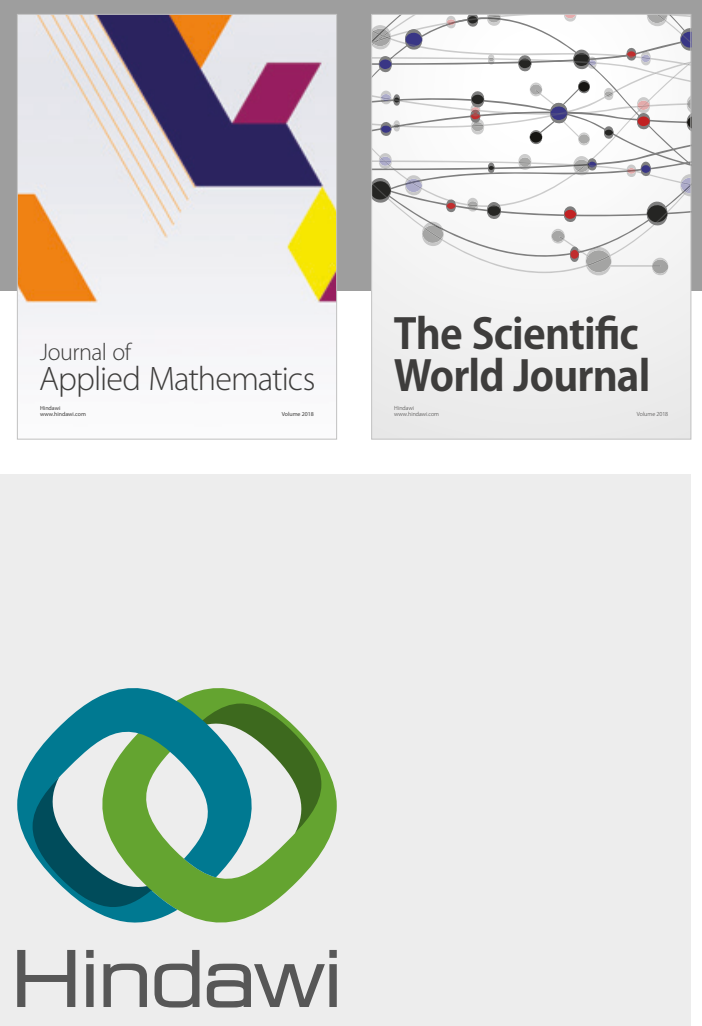

Submit your manuscripts at

www.hindawi.com

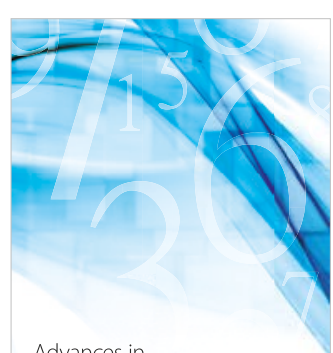

Advances in
Numerical Analysis
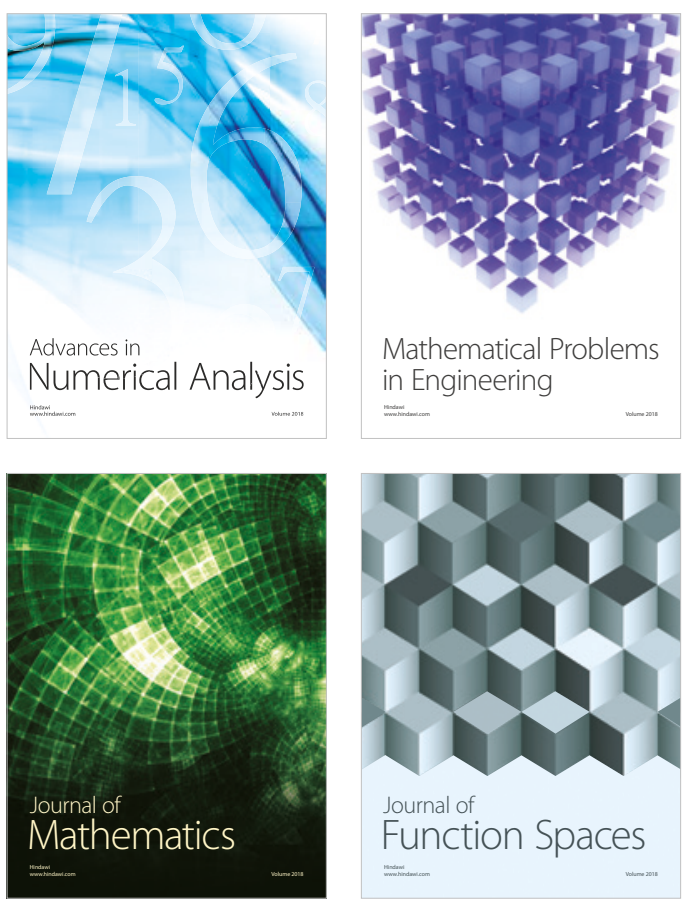

Mathematical Problems in Engineering

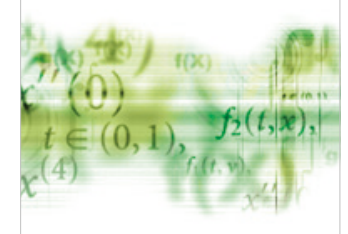

International Journal of

Differential Equations

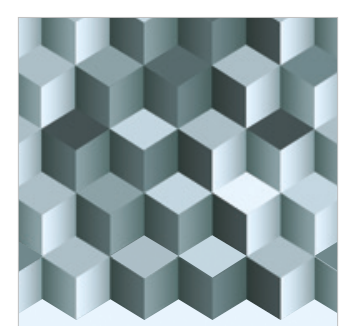

Journal of

Function Spaces

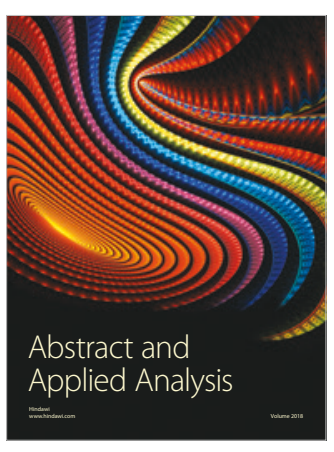

The Scientific

World Journal

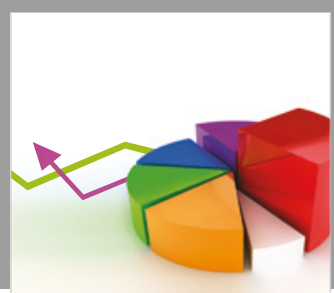

Journal of

Probability and Statistics
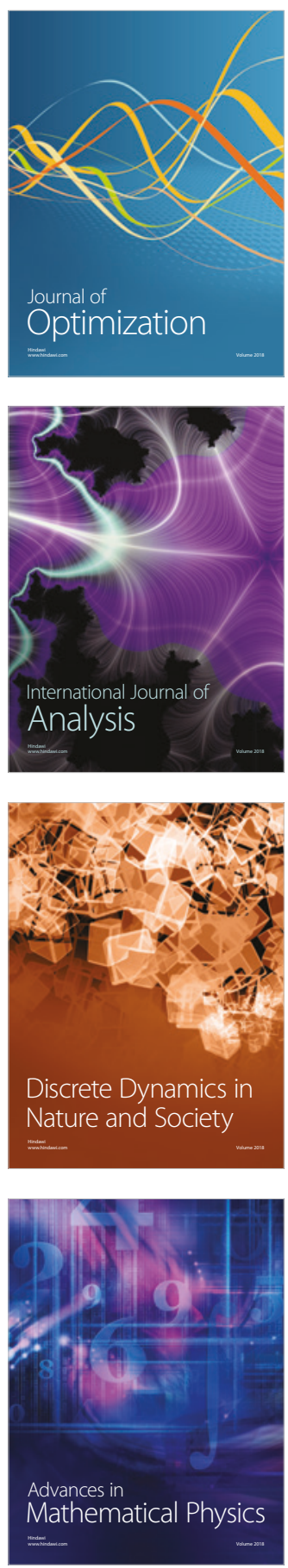